Bull. Korean Math. Soc. 52 (2015), No. 2, pp. 505-511

http://dx.doi.org/10.4134/BKMS.2015.52.2.505

\title{
SOME UNIFORM GEOMETRICAL PROPERTIES IN BANACH SPACES
}

\author{
Kyugeun Cho and Chongsung Lee
}

ABSTRACT. In this paper, we investigate relationships among property $(k-\beta)$, weak property $\left(\beta_{k}\right), k$-nearly uniformly convexity and property $\left(A_{k}\right)$.

\section{Introduction}

Let $(X,\|\cdot\|)$ be a real Banach space and $X^{*}$ the dual space of $X$. By $B_{X}$ and $S_{X}$, we denote the closed unit ball of $X$ and the unit sphere of $X$, respectively. For a sequence $\left(x_{n}\right)$ in $X$, we let $\operatorname{sep}\left(x_{n}\right)=\inf \left\{\left\|x_{n}-x_{m}\right\|: n \neq m\right\}$. Denote by $\mathbb{N}$ and $\mathbb{R}$ the set of natural numbers and real numbers, respectively. For $A, B \subset \mathbb{N}$, we write $A<B$ if $\max A<\min B$. For a Banach space $X$ with a basis $\left(e_{n}\right)$ and $x, y \in X$, we write $x<y$ if supp $x<\operatorname{supp} y$, where $\operatorname{supp} x=\left\{i \in \mathbb{N}: a_{i} \neq 0, x=\sum_{i=1}^{\infty} a_{i} e_{i}\right\}$.

A Banach space is said to be reflexive if the natural embedding $\eta: X \rightarrow X^{* *}$ is onto. A Banach space $X$ is said to have Banach-Saks property (BS) if any bounded sequence in the space admits a subsequence whose arithmetic means converge in norm. In similar way, we say that a Banach space $X$ has weak Banach-Saks property (w-BS) if any weakly convergent sequence in the space admits a subsequence whose arithmetic means converge in norm. Since any weakly convergent sequence is norm bounded, it follows that Banach-Saks property implies weak Banach-Saks property. We note that weak Banach-Saks property and Banach-Saks property coincide in the reflexive Banach space. A Banach space $X$ is said to be uniformly convex (UC) if for every $\epsilon>0$, there exists $\delta>0$ such that if $x, y \in B_{X}$ and $\|x-y\| \geq \epsilon, \frac{1}{2}\|x+y\| \leq 1-\delta$.

S. Kakutani [2] showed that unform convexity implies Banach-Saks property. T. Nishiura and D. Waterman [6] proved that Banach-Saks property implies reflexivity in Banach spaces.

Received January 17, 2014; Revised May 8, 2014.

2010 Mathematics Subject Classification. 46B20.

Key words and phrases. property $(k-\beta)$, weak property $\left(\beta_{k}\right), k$-nearly uniformly convexity, property $\left(A_{k}\right)$

This work was supported by the Inha University Research Grant. 
A Banach space is said to have property $\left(A_{k}\right)$ if it is reflexive and there exists a number $\delta, 0<\delta<1$, such that for a weakly null sequence $\left(x_{n}\right)$, there exist $n_{1}<n_{2}<\cdots<n_{k}$ with $\left\|\frac{1}{k} \sum_{i=1}^{k} x_{n_{i}}\right\| \leq 1-\delta$. We say that $X$ has the property $\left(A_{\infty}\right)$ if it has the property $\left(A_{k}\right)$ for some $k \in \mathbb{N}$.

Let $x_{1}, x_{2}, \ldots, x_{k+1} \in X$. The $k$-dimensional volume enclosed by $x_{1}, x_{2}, \ldots$, $x_{k+1}$ is given by

$$
\begin{aligned}
& V\left(x_{1}, x_{2}, \ldots, x_{k+1}\right) \\
= & \sup \left\{\left|\begin{array}{ccc}
1 & \cdots & 1 \\
f_{1}\left(x_{1}\right) & \cdots & f_{1}\left(x_{k+1}\right) \\
\vdots & & \vdots \\
f_{k}\left(x_{1}\right) & \cdots & f_{k}\left(x_{k+1}\right)
\end{array}\right| f_{i} \in B_{X^{*}}, i=1,2, \ldots, k\right\} .
\end{aligned}
$$

A Banach space $X$ is said to be $k$-uniformly rotund ( $k$-UR), $k \geq 1$, if for each $\epsilon>0$ there exists a $\delta>0$ such that for $x_{i} \in B_{X}(i=1,2, \ldots, k+1)$ and $V\left(x_{1}, x_{2}, \ldots, x_{k+1}\right) \geq \epsilon, \frac{1}{k+1}\left\|\sum_{i=1}^{k+1} x_{i}\right\| \leq 1-\delta$.

J. R. Partington [7] introduced the notion of property $\left(A_{k}\right)$ and show the following strict implications:

$$
(U C) \Rightarrow\left(A_{2}\right) \Rightarrow\left(A_{3}\right) \Rightarrow \cdots \Rightarrow\left(A_{\infty}\right) \Rightarrow(B S)
$$

A Banach space $X$ is said to have the property $(k-\beta)$ if for each $\epsilon>0$, there exists $\delta>0$ such that for any $x \in B_{X}$ and any sequence $\left(x_{n}\right) \subset B_{X}$ with $\operatorname{sep}\left(x_{n}\right)>\epsilon$ there exist $n_{i} \in \mathbb{N}, i=1,2, \ldots, k$ with $n_{1}<n_{2}<\cdots<n_{k}$ such that

$$
\left\|\frac{1}{k+1}\left(x+\sum_{i=1}^{k} x_{n_{i}}\right)\right\| \leq 1-\delta .
$$

A Banach space $X$ is said to be $k$-nearly uniformly convex $(k-N U C)$ if for each $\epsilon>0$, there exists $\delta>0$ such that for any sequence $\left(x_{n}\right) \subset B_{X}$ with $\operatorname{sep}\left(x_{n}\right)>\epsilon$ there exist $n_{i} \in \mathbb{N}, i=1,2, \ldots, k$ with $n_{1}<n_{2}<\cdots<n_{k}$ such that

$$
\left\|\frac{1}{k}\left(\sum_{i=1}^{k} x_{n_{i}}\right)\right\| \leq 1-\delta .
$$

D. Kutzarova [3] introduced the notion of property $(k-\beta)$ and $k$-nearly uniformly convexity and show the following strict implications:

$$
\begin{aligned}
& (U C) \Rightarrow\left(\beta_{1}\right) \Rightarrow 2-N U C \Rightarrow\left(\beta_{2}\right) \Rightarrow 3-N U C \Rightarrow \cdots \Rightarrow(B S) \\
& \Downarrow \\
& A_{2} \quad A_{3}
\end{aligned}
$$

A Banach space $X$ has the weak property $\left(\beta_{k}\right)$ if it is reflexive and there exists $\delta>0$ such that for any $x \in B_{X}$ and any weakly null sequence $\left(x_{n}\right) \subset B_{X}$ there 
exist $n_{i} \in \mathbb{N}, i=1,2, \ldots, k$ with $n_{1}<n_{2}<\cdots<n_{k}$ such that

$$
\left\|\frac{1}{k+1}\left(x+\sum_{i=1}^{k} x_{n_{i}}\right)\right\| \leq 1-\delta .
$$

We say that $X$ has the weak property $\left(\beta_{\infty}\right)$ if it has the weak property $\left(\beta_{k}\right)$ for some $k \in \mathbb{N}$. K. G. Cho and C. S. Lee [1] introduced the notion of weak property $\left(\beta_{k}\right)$ and show the following strict implications:

$$
(U C) \Rightarrow\left(w-\beta_{1}\right) \Rightarrow\left(w-\beta_{2}\right) \Rightarrow \cdots \Rightarrow\left(w-\beta_{\infty}\right) \Rightarrow(B S)
$$

In this paper, we show that the property $(k-\beta)$ implies the weak property $\left(\beta_{k}\right)$ but the converse does not hold.

\section{Main parts}

We begin with the proposition:

Proposition 2.1. Let $X$ be a Banach space.

(1) If $X$ has the weak property $\left(\beta_{k}\right)$, then it has the property $\left(A_{k+1}\right)$ for all $k \geq 1$.

(2) If $X$ has the property $\left(A_{k}\right)$, then it has the weak property $\left(\beta_{k}\right)$ for all $k \geq 2$.

Proof. (1) Choose a corresponding $\delta>0$ according to the definition of weak property $\left(\beta_{k}\right)$. Let $\left(x_{n}\right)$ be a weakly null sequence in $B_{X}$. Take $x=x_{1}=x_{n_{1}}$, then for $\left(x_{n}\right)_{n \geq 2}$ there exist $2 \leq n_{2}<n_{3}<\cdots<n_{k+1}$ such that

$$
\frac{1}{k+1}\left\|\sum_{i=1}^{k+1} x_{n_{i}}\right\|=\frac{1}{k+1}\left\|x+\sum_{i=2}^{k+1} x_{n_{i}}\right\| \leq 1-\delta .
$$

This means that $X$ has the property $\left(A_{k+1}\right)$.

(2) Choose a corresponding $\delta>0$ according to the definition of property $\left(A_{k}\right)$. Let $x \in B_{X}$ and $\left(x_{n}\right)$ be a weakly null sequence in $B_{X}$. Since $X$ has the property $\left(A_{k}\right)$, there exist $n_{1}<n_{2}<\cdots<n_{k}$ such that

$$
\frac{1}{k}\left\|\sum_{i=1}^{k} x_{n_{i}}\right\| \leq 1-\delta
$$

Then

$$
\frac{1}{k+1}\left\|x+\sum_{i=1}^{k} x_{n_{i}}\right\| \leq \frac{1}{k+1}(1+k(1-\delta))=1-\frac{k \delta}{k+1}
$$

Taking $\delta_{1}=\frac{k \delta}{k+1}$, we get the result.

We want to show the converses of Proposition 2.1 do not hold. We need lemma, example and proposition. The following lemma and example can be found in [4] and [7], respectively. 
Lemma 2.2. $l_{1}$-direct sum of finitely many Banach spaces with $k$-NUC has $k-N U C$.

Example 1. For $x=\left(a_{n}\right) \in l_{2}$, we define a norm $\|x\|_{(k)}$ by

$$
\|x\|_{(k)}=\left[\sup _{n_{1}<n_{2}<\cdots<n_{k}}\left(\sum_{i=1}^{k}\left|a_{n_{i}}\right|\right)^{2}+\sum_{n \neq n_{1}, n_{2}, \ldots, n_{k}}\left|a_{n}\right|^{2}\right]^{\frac{1}{2}} .
$$

Then $\|x\|_{2} \leq\|x\|_{(k)} \leq \sqrt{k}\|x\|_{2}$. Let $X_{k}=\left(l_{2},\|\cdot\|_{(k)}\right)$. Then $X_{k}$ has the property $\left(A_{k+1}\right)$ but not the property $\left(A_{k}\right)[7]$.

Proposition 2.3. The property $(k-\beta)$ implies the weak property $\beta_{k}$.

Proof. Let $\delta\left(\frac{1}{2}\right)$ be chosen according to the definition of $(k-\beta)$ for $\epsilon=\frac{1}{2}$. Put $\delta=\min \left\{\frac{k}{2(k+1)}, \delta\left(\frac{1}{2}\right)\right\}$. Let $x \in B_{X}$ and $\left(x_{n}\right)$ be a weakly null sequence in $B_{X}$. If there exist $n_{1}<n_{2}<\cdots<n_{k}$ such that $\left\|x_{n_{i}}\right\| \leq \frac{1}{2}$, then $\left\|\frac{1}{k+1}\left(x+\sum_{i=1}^{k} x_{n_{i}}\right)\right\| \leq \frac{1}{k+1}\left(1+\frac{k}{2}\right)=1-\frac{k}{2(k+1)} \leq 1-\delta$. We now consider the case that $\left\|x_{n}\right\|>\frac{1}{2}$ except finite $n$ less than $k$. Assume $\left\|x_{n}\right\|>\frac{1}{2}$ for all $n \in \mathbb{N}$. We note that $\liminf _{n \rightarrow \infty}\left\|x_{n}-x_{m}\right\| \geq\left\|x_{m}\right\|>\frac{1}{2}$ for every $m$. Put $n_{1}=1$. Since $\liminf \inf _{n \rightarrow \infty}\left\|x_{n}-x_{1}\right\| \geq\left\|x_{1}\right\|>\frac{1}{2}$, there exists $n_{2}$ with $n_{2}>n_{1}$ such that $\left\|x_{n_{1}}-x_{n_{2}}\right\|>\frac{1}{2}$. Having chosen $x_{n_{1}}, \ldots, x_{n_{l}}$ with $\left\|x_{n_{i}}-x_{n_{j}}\right\|>\frac{1}{2}$ whenever $i \neq j, 1 \leq i, j \leq l$. Since $\liminf _{n \rightarrow \infty}\left\|x_{n}-x_{n_{i}}\right\| \geq\left\|x_{n_{i}}\right\|>\frac{1}{2}$ for $i=1,2, \ldots, l$, there exists $n_{l+1}$ with $n_{l+1}>n_{l}$ such that $\left\|x_{n_{i}}-x_{n_{l+1}}\right\|>\frac{1}{2}$ for $i=1,2, \ldots, l$.

We get a subsequence $\left(x_{n_{i}}\right)$ of $\left(x_{n}\right)$ with $\operatorname{sep}\left(x_{n_{i}}\right) \geq \frac{1}{2}$. Then there exist $x_{n_{i_{1}}}, \ldots, x_{n_{i_{k}}}$ such that

$$
\frac{1}{k+1}\left\|x+\sum_{j=1}^{k} x_{n_{i_{j}}}\right\| \leq \delta\left(\frac{1}{2}\right) \leq 1-\delta .
$$

This completes the proof.

We are ready to show that the converses of Proposition 2.1 do not hold. The techniques are same with [3].

Proposition 2.4. (1) There exists a Banach space with the property $\left(A_{k+1}\right)$ which does not have the weak property $\left(\beta_{k}\right)$ for each $k \geq 1$.

(2) There exists a Banach space with the weak property $\left(\beta_{k}\right)$ which does not have the property $\left(A_{k}\right)$ for each $k \geq 2$.

Proof. (1) Since $l_{2}$ is uniformly convex, it is 2 -NUC. By Lemma $2.2,\left(l_{2}+\mathbb{R}\right)_{l_{1}}$ is 2 -NUC. Since 2 -NUC implies the property $\left(A_{2}\right)[3],\left(l_{2}+\mathbb{R}\right)_{l_{1}}$ has the property $\left(A_{2}\right)$. Let $\left(e_{n}\right)$ be the usual unit bases of $l_{2}$. Then $\left(\left(e_{n}, 0\right)\right)$ is weakly null in $\left(l_{2}+\right.$ $\mathbb{R})_{l_{1}}$. But for $(0,1) \in\left(l_{2}+\mathbb{R}\right)_{l_{1}},\left\|(0,1)+\left(e_{n}, 0\right)\right\|_{\left(l_{2}+\mathbb{R}\right)_{l_{1}}}=\left\|\left(e_{n}, 1\right)\right\|_{\left(l_{2}+\mathbb{R}\right)_{l_{1}}}=2$. This means that $\left(l_{2}+\mathbb{R}\right)_{l_{1}}$ does not have the weak property $\left(\beta_{1}\right)$. 
For $k \geq 2, X_{k}$ is $k$-UR [5]. Since $k$-UR implies $(k-\beta)$ [3], $X_{k}$ is $(k-\beta)$. Since $(k-\beta)$ implies $(k+1)$-NUC [3], $X_{k}$ is $(k+1)$-NUC. By Lemma $2.2,\left(X_{k}+\mathbb{R}\right)_{l_{1}}$ is $(k+1)$-NUC. Since $(k+1)$-NUC implies the property $A_{k+1}[3],\left(X_{k}+\mathbb{R}\right)_{l_{1}}$ has the property $A_{k+1}$.

We note that $X_{k}$ does not have the property $A_{k}$ [7]. Then for all $\delta>0$, there exists weakly null sequence $\left(x_{n}(\delta)\right)$ in $B_{X_{k}}$ such that $\frac{1}{k}\left\|\sum_{i=1}^{k} x_{n_{i}}(\delta)\right\|_{(k)}>1-\delta$ for all $n_{1}<n_{2}<\cdots<n_{k}$. Let $y=(0,1), y_{n}=\left(x_{n}(\delta), 0\right)$. Then $y, y_{n} \in$ $B_{\left(X_{k}+\mathbb{R}\right)_{l_{1}}},\left(y_{n}\right)$ is weakly null in $\left(X_{k}+\mathbb{R}\right)_{l_{1}}$ and

$$
\begin{aligned}
\frac{1}{k+1}\left\|y+\sum_{i=1}^{k} y_{n_{i}}\right\|_{\left(X_{k}+\mathbb{R}\right)_{l_{1}}} & =\frac{1}{k+1}\left(1+\left\|\sum_{i=1}^{k} x_{n_{i}}(\delta)\right\|_{(k)}\right) \\
& \geq \frac{1}{k+1}(1+k(1-\delta))=1-\frac{k}{k+1} \delta
\end{aligned}
$$

for all $n_{1}<n_{2}<\cdots<n_{k}$. This means that $\left(X_{k}+\mathbb{R}\right)_{l_{1}}$ does not have the weak property $\left(\beta_{k}\right)$.

(2) $X_{k}$ does not have the property $A_{k}$ [7] and is $k$-UR [5]. Since $k$-UR implies $(k-\beta)[3], X_{k}$ is $(k-\beta)$. By Proposition $2.3, X_{k}$ has the weak property $\beta_{k}$. This completes the proof.

We finally investigate the converse of Proposition 2.3.

Example 2. Let $X=\left(\prod_{p \geq 2} l_{p}\right)_{2}$ and $\left(e_{n}^{(p)}\right)$ be a usual unit basis for $l_{p}$. For $n=0,1,2, \ldots$ and $1 \leq p \leq n+1$, let $\frac{f_{n(n+1)}+p}{2}=\left(0, \ldots, 0, e_{n-p+2}^{(p+1)}, 0, \ldots\right)$ with all zero except $p$ th coordinate $e_{n-p+2}^{(p+1)}$. Then $\left(f_{i}\right)$ is a basis for $X=\left(\prod_{p \geq 2} l_{p}\right)_{2}$.

We need a lemma.

Lemma 2.5. Let $x, y \in B_{\left(\prod_{p \geq 2} l_{p}\right)_{2}}$ with $x<y$. Then $\|x+y\| \leq \sqrt{2}$.

Proof. Let $x=\sum_{i=1}^{n} a_{i} f_{i}$ and $y=\sum_{i=n+1}^{\infty} a_{i} f_{i}$. For $p \geq 2$, let

$$
A_{p}=\left\{i \in \operatorname{supp} x: i=\frac{m(m+1)}{2}+p \text { for } m \geq 0\right\}
$$

and

$$
B_{p}=\left\{i \in \operatorname{supp} y: i=\frac{m(m+1)}{2}+p \text { for } m \geq 0\right\} .
$$

Then for all $p \geq 2$, we know $A_{p}<B_{p}$ and

$$
\|x+y\|^{2}=\sum_{p=2}^{\infty}\left\|\sum_{i \in A_{p}} a_{i} e_{i}^{(p)}+\sum_{i \in B_{p}} a_{i} e_{i}^{(p)}\right\|_{p}^{2}
$$




$$
\begin{aligned}
& =\sum_{p=2}^{\infty}\left(\sum_{i \in A_{p} \cup B_{p}}\left|a_{i}\right|^{p}\right)^{\frac{2}{p}} \\
& \leq \sum_{p=2}^{\infty}\left(\sum_{i \in A_{p}}\left|a_{i}\right|^{p}\right)^{\frac{2}{p}}+\sum_{p=2}^{\infty}\left(\sum_{i \in B_{p}}\left|a_{i}\right|^{p}\right)^{\frac{2}{p}} \\
& =\|x\|^{2}+\|y\|^{2}=2 .
\end{aligned}
$$

This completes the proof.

Using the above lemma, we get the following.

Proposition 2.6. There exists a Banach space with the weak property $\left(\beta_{1}\right)$ which is not $k$-NUC for all $k \geq 2$.

Proof. Let $X=\left(\prod_{p \geq 2} l_{p}\right)_{2}$. Let $x=\sum_{i=1}^{\infty} a_{i} f_{i}, x_{n}=\sum_{i=1}^{\infty} b_{i}^{(n)} f_{i} \in B_{X}$ and $\left(x_{n}\right)$ be a weakly null sequence in $B_{X}$. Then there exists $N \in \mathbb{N}$ such that $\left\|\sum_{i=N+1}^{\infty} a_{i} f_{i}\right\| \leq \frac{1}{4}$. Since $\left(x_{n}\right)$ be a weakly null sequence in $B_{X}$, there exists $n_{0} \in \mathbb{N}$ such that $\left\|\sum_{i=1}^{N} b_{i}^{\left(n_{0}\right)} f_{i}\right\| \leq \frac{1}{4}$. Then by Lemma 2.5,

$$
\begin{aligned}
\left\|x+x_{n_{0}}\right\| & \leq\left\|\sum_{i \leq N} a_{i} f_{i}+\sum_{i \geq N+1} b_{i}^{\left(n_{0}\right)} f_{i}\right\|+\left\|\sum_{i=N+1}^{\infty} a_{i} f_{i}\right\|+\left\|\sum_{i=1}^{N} b_{i}^{\left(n_{0}\right)} f_{i}\right\| \\
& \leq \sqrt{2}+\frac{1}{2}=2\left(1-\left(\frac{3}{4}-\frac{\sqrt{2}}{2}\right)\right) .
\end{aligned}
$$

Taking $\delta=\frac{3}{4}-\frac{\sqrt{2}}{2}, X=\left(\prod_{p \geq 2} l_{p}\right)_{2}$ has the weak property $\left(\beta_{1}\right)$. On the other hand, $X$ fails to have an equivalent NUC norm [3]. We get the result.

The above proposition also implies that there exists a space which satisfies the weak property $\left(\beta_{1}\right)$ but does not satisfy the property $(k-\beta)$ for all $k$. By [1], [3], [7], Propositions 2.1, 2.3, 2.4 and 2.6, we finally get the following strict implications:

$$
\begin{aligned}
& (U C) \Rightarrow\left(\beta_{1}\right) \Rightarrow 2-N U C \Rightarrow\left(\beta_{2}\right) \Rightarrow 3-N U C \Rightarrow \cdots \Rightarrow(B S) \\
& \Downarrow \quad \Downarrow \quad \forall \quad \forall \quad \cdots \\
& (U C) \Rightarrow\left(w-\beta_{1}\right) \Rightarrow\left(A_{2}\right) \Rightarrow\left(w-\beta_{2}\right) \Rightarrow\left(A_{3}\right) \Rightarrow \cdots \Rightarrow(B S)
\end{aligned}
$$

Acknowledgment. We are grateful to the referee for the careful reading of the manuscript and very useful comments. 


\section{References}

[1] K. G. Cho and C. S. Lee, Weak property $\left(\beta_{k}\right)$, Korean J. Math. 20 (2012), no. 4, 415-422.

[2] S. Kakutani, Weak convergence in uniformly convex spaces, Tôhoku Math. J. 45 (1938), 347-354.

[3] D. Kutzarova, $k$-beta and $k$-Nearly Uniformly Convex Banach spaces, J. Math. Anal. Appl. 162 (1991), no. 2, 322-338.

[4] D. Kutzarova and T. Landes, NUC and related properties of direct sums, Bolletino U.M.I.(7)8-A (1994), 45-54.

[5] B.-L. Lin and X.-Y. Yu, On the $k$-uniform rotund and the fully convex Banach spaces, J. Math. Anal. Appl. 110 (1985), no. 2, 407-410.

[6] T. Nishiura and D. Waterman, Reflexivity and summability, Studia Math. 23 (1963), 53-57.

[7] J. R. Partington, On the Banach-Saks property, Math. Proc. Cambridge Philos. Soc. 82 (1977), no. 3, 369-374.

\section{KYugEun CHO}

Bangmok College of General Education

Myong Ji University

YONG-IN 449-728, KOREA

E-mail address: kgjo@mju.ac.kr

Chongsung Lee

Department of Mathematics education

INHA UNIVERSITY

INCHON 402-751, KOREA

E-mail address: cslee@inha.ac.kr 